\title{
Fundamental Contribution of $\beta$-Oxidation to Polyketide Mycotoxin Production In Planta
}

\author{
Lori A. Maggio-Hall, Richard A. Wilson, and Nancy P. Keller \\ Department of Plant Pathology, University of Wisconsin-Madison, 1630 Linden Drive, Madison, WI 53706, U.S.A.
}

Submitted 31 January 2005. Accepted 28 March 2005.

\begin{abstract}
Seed contamination with polyketide mycotoxins, including aflatoxin (AF) and sterigmatocystin (ST) produced by $A s-$ pergillus spp., is an agricultural, economic, and medical issue worldwide. Acetyl-CoA, the fundamental building block of all known fungal polyketides, is generated by a large number of biochemical pathways, including $\beta$-oxidation of fatty acids and glycolysis of sugars. We present several lines of evidence to support a major role for seed fatty acids in formation of AF and ST in A. flavus, A. parasiticus, and $A$. nidulans. Aspergillus strains exhibiting canonical signs of oleic acid-induced peroxisome proliferation, including increased catalase activity, $\beta$-oxidation gene expression, and peroxisomal clustering, also exhibited a marked increase in toxin gene expression and biosynthesis. Furthermore, microscopic observations showed that the ST and AF precursor norsolorinic acid accumulated in peroxisomes of all three Aspergilli. While a peroxisomal $\beta$ oxidation mutation eliminated oleic acid-induced increases in ST in A. nidulans, a mitochondrial $\beta$-oxidation mutation played a larger role in eliminating ST formation on oatmeal medium and on live corn kernels, implicating a fundamental role for both peroxisomal and mitochondrial $\beta$ oxidation in toxin production.
\end{abstract}

Agricultural fungal toxins that cause animal illnesses, exemplified by the discovery of aflatoxin as causal agent of the infamous Turkey X disease (Hicks et al. 2002; Lancaster et al. 1961), are referred to as mycotoxins, and some mycotoxins, such as the Fusarium trichothecenes, can also be virulence factors (Proctor et al. 1995). Although there has been significant progress in the last decade in identifying and characterizing the biosynthetic genes involved in fungal toxin biosynthesisleading to such remarkable observations as the frequent organization of these genes in clusters (Keller and Hohn 1996), very little is known about induction and regulation of either toxin gene expression or toxin formation in planta.

Many fungal toxins are members of a large, diverse class of compounds known collectively as polyketides. Polyketides interact with living organisms in multifarious ways, and conse-

\section{A. Maggio-Hall and R. A. Wilson made equal contributions.}

Corresponding author: N. P. Keller; Telephone: +1.608 .262 .9795 . Fax: +1.608.263.2626; E-mail: npk@ plantpath.wisc.edu

Current address for R. A. Wilson: School of Biological Sciences, University of Exeter, Washington Singer Laboratories, Perry Road, Exeter, Devon EX4 4QG, U.K.

Current address for L. A. Maggio-Hall: I.E. DuPont de Nemours and Company, Central Research and Development, Biochemical Science and Engineering Experimental Station, Wilmington, DE 19880, U.S.A. quently, much is now understood about the mechanisms of polyketide biosynthesis (Hopwood and Sherman 1990). Acetyl-CoA, both as polyketide starter unit and extender unit via malonylCoA formation, is the fundamental building block of most fungal, plant, and bacterial polyketides. While involved in secondary metabolism, acetyl-CoA truly lies at the heart of primary metabolism. Virtually all known carbon sources are metabolized via acetyl-CoA. It is oxidized to $\mathrm{CO}_{2}$ by the tricarboxylic acid cycle, thus serving as the primary source of electrons for ATP synthesis for many organisms, eukaryotic and prokaryotic. Moreover, it is an essential intermediate in the biosynthesis of membrane and storage lipids, a process that, like polyketide synthesis, requires conversion of acetyl-CoA to malonyl-CoA. Knowledge of which carbon sources contribute most directly or efficiently to the acetyl-CoA pool available for polyketide biosynthesis could lead to enhanced production of beneficial compounds such as antibiotics and anticancer drugs or, conversely, to strategies to eliminate harmful polyketides such as those associated with agricultural commodities.

Arguably, the most intensively examined polyketide toxin with respect to gene and nutrient regulation is aflatoxin (AF), a mycotoxin produced by Aspergillus parasiticus and A. flavus, common pathogens of oilseed crops, including corn and peanuts. The vast economic losses, up to $\$ 100$ million per year in the United States (Robens August 2001), and health costs of AF contamination, including sporadic deaths from acute, highlevel exposure and liver cancer from chronic, low-level exposure (Bosch and Peers 1991; Centers for Disease Control 2004; Wogan et al. 1992), have led to the establishment of national and international standards governing allowable contamination levels of AF in agricultural commodities. Much of our understanding of the molecular genetics of AF regulation comes from studies of sterigmatocystin (ST) biosynthesis in the model system A. nidulans (Hicks et al. 2002). ST, the penultimate intermediate in AF biosynthesis and the end product of the pathway for a number of Aspergilli and other genera (Barnes et al. 1994), has similar cytotoxic and carcinogenic properties to AF but is not a major agricultural contaminant (Purchase and van der Watt 1970). Recent genetic and biochemical studies of ST biosynthesis in A. nidulans showed that alterations in acyl-CoA ratios, in particular increased propionyl-CoA to acetyl-CoA concentrations, could block ST biosynthesis despite active ST gene expression (Zhang and Keller 2004). This study highlighted the importance of accessibility of cellular pools of acetyl-CoA to polyketide metabolism. Logic therefore suggests that metabolic pathways that contribute to the acetyl-CoA pool, such as fatty acid $\beta$-oxidation or glycolysis of sugars, are important in generating polyketide starter units.

Historically, studies of AF production have focused on the apparent regulation of AF biosynthesis by glucose (Abdollahi and Buchanan 1981; Buchanan and Lewis 1984; Buchanan et 
al. 1987; Davis and Diener 1968; Wiseman and Buchanan 1987), a sugar contributing to the acetyl-CoA pool via glycolysis. Some of the highest yields of AF are reported from $A$. flavus and A. parasiticus strains grown on this carbon source, and increases in glucose concentration boost ST biosynthesis in $A$. nidulans (N. P. Keller, unpublished data). The glucose-mediated regulatory mechanism remains unknown but likely responds to cAMP levels (Roze et al. 2004). Although the majority of laboratory studies have investigated the influence of glucose and other simple sugars on AF biosynthesis, higher incidences of AF contamination in the field or storage milieus are associated with high-oil or high-oleic varieties of corn and peanut (Severns et al. 2003; Xue et al. 2003). Furthermore Aspergillus growth and toxin production in corn appears to occur primarily in the embryo and aleurone layer (Brown et al. 1993; Keller et al. 1994; Smart et al. 1990), which are known to house the majority of seed lipids in this plant (Watson 1987).

Laboratory studies of AF yield from strains grown on laboratory media containing fatty acids appear inconsistent, with some papers reporting low or no AF production (Davis and Diener 1968), while others report increases in AF production compared with media without added fatty acids (Burow et al. 1997; Fanelli et al. 1983). Careful examination of these studies, however, indicates that these differences in AF production might lie in the ingredients of the lipid-based growth medium. First, the composition of available oxygenated fatty acids, known as oxylipins, can have a vast influence on AF production with some oxylipins stimulating and some inhibiting AF production (Burow et al. 1997; Fabbri et al. 1983; Fanelli et al. 1983; Goodrich-Tanrikulu et al. 1995). Secondly, a mixture of lipids and sugars appears important in AF production during vegetative growth in media. For example, cottonseed oil as a sole carbon source was shown to support only a small amount of AF production, but removal of oil from ground whole cotton seeds decreased AF production on this defatted seed by 800 fold (Mellon et al. 2000). This demonstrates that lipids are necessary but not sufficient for optimal AF biosynthesis, suggesting both lipids and sugars contribute to regulation of carbon and its flow into the ST and AF pathway.

Table 1. List of strains

\begin{tabular}{|c|c|c|}
\hline Strain & Genotype & Source \\
\hline \multicolumn{3}{|c|}{ Aspergillus nidulans strains } \\
\hline A 26 & biA1; veA1 & FGSC $^{a}$ \\
\hline RLMH12 & biA $1 ;$ met $G 1 ; \triangle$ foxA::met $G ;$ veA 1 & $\begin{array}{l}\text { Maggio-Hall and } \\
\text { Keller } 2004\end{array}$ \\
\hline RLMH14 & $\begin{array}{l}\text { biA } 1 ; \arg B 2 ; \operatorname{met} G 1 ; \triangle \text { foxA::met } G \\
\quad \Delta o d e A:: \arg B ; \text { veA } 1\end{array}$ & This study \\
\hline RLMH41 & $\begin{array}{l}\text { biA } 1 ; \text { pyrG89; } \Delta e c h A:: p y r G^{\text {A.f. }} \\
\text { veA } 1\end{array}$ & $\begin{array}{l}\text { Maggio-Hall and } \\
\text { Keller } 2004\end{array}$ \\
\hline RRAW10.4 & $\begin{array}{l}\text { biA1; acuD ::gfp; } \triangle \text { odeA::argB; } \\
\quad \text { veA1 }\end{array}$ & This study \\
\hline RRAW 10.5 & $\begin{array}{l}\text { biA1; } \Delta s t c E:: \arg B ; a c u D:: g f p ; \\
\quad v e A 1\end{array}$ & This study \\
\hline RRAW14.2 & biA $1 ; a c u D:: g f p ; v e A 1$ & $\begin{array}{l}\text { Maggio-Hall and } \\
\text { Keller } 2004\end{array}$ \\
\hline TAMC31.65 & biA $1 ; \arg B 2 ; \triangle$ odeA::argB $;$ veA1 & Calvo et al. 2001 \\
\hline TLMH7 & $\begin{array}{l}\text { biA } 1 ; \text { pyrG89; } \Delta e c h A:: p y r G^{\text {A.f }} \\
\text { met } G 1 ; \Delta \text { foxA }:: \text { met } G ; \text { veA } 1\end{array}$ & $\begin{array}{l}\text { Maggio-Hall and } \\
\text { Keller } 2004\end{array}$ \\
\hline \multicolumn{3}{|l|}{ A. flavus strains } \\
\hline $12 \mathrm{~S}$ & wild type & P. Cotty \\
\hline MAEW4 & norA & This study \\
\hline \multicolumn{3}{|c|}{ A. parasiticus strains } \\
\hline SRRC 126 & norA & $\mathrm{SRRC}^{\mathrm{b}}$ \\
\hline ATCC 36537 & verA; $w A$ & $\mathrm{ATCC}^{\mathrm{c}}$ \\
\hline TAMC 37.41 & verA; $\triangle$ ode $A ; w A$ & Wilson et al. 2004 \\
\hline
\end{tabular}

${ }^{\text {a }}$ Fungal Genetics Stock Center, Kansas City, MO, U.S.A.

${ }^{\mathrm{b}}$ Southern Regional Research Center, USDA, New Orleans.

${ }^{c}$ American Type Culture Collection, Manassas, VA, U.S.A.
We have taken a closer look at the effect of fatty-acid metabolism, a potential source of acetyl-CoA through $\beta$-oxidation, on synthesis of ST and AF both on laboratory growth media and in planta. Through a combination of genetic, biochemical, and microscopic studies using wild type and Aspergillus fatty acid-metabolism mutants, we present several lines of evidence to support a fundamental role for fatty acids in both transcriptional regulation of ST and AF genes and in supplying acetyl$\mathrm{CoA}$, via $\beta$-oxidation, for toxin biosynthesis. Both peroxisomal and mitochondrial $\beta$-oxidation pathways are involved in this process. Furthermore, whereas the $\beta$-oxidation contribution to toxin production can be bypassed by sugar content in media, it appears to be essential in planta.

\section{RESULTS}

Increased ST and AF gene transcription and toxin accumulation in oleate desaturase mutants.

Previous studies to investigate the role of lipid metabolism in Aspergillus development generated deletions of the $\Delta-12$ (oleate) desaturase (odeA) gene in A. nidulans (Calvo et al. 2001) and $A$. parasiticus (Wilson et al. 2004). Both $\Delta o d e A$ strains (Table 1, TAMC31.65 and TAMC37.41) showed significant accumulation of oleic acid and increased total fattyacid content (Calvo et al. 2001; Wilson et al. 2004). Liquid chromatography/mass spectrometry (LC/MS) analysis showed the A. nidulans $\Delta o d e A$ strain to have significantly higher ST accumulation on glucose medium (280 micrograms of ST per gram of fungal biomass) than wild type (A26, $193 \mu \mathrm{g} / \mathrm{g}$ ), as exemplified in Figure 1A, and concomitant increased expression of an ST biosynthetic gene, stcU (Fig. 1B). Previous studies have found expression of stc $U$ to be representative of the expression of most other cluster genes (Brown et al. 1996b; Shimizu and Keller 2001). The A. parasiticus $\Delta o d e A$ allele, placed in an AF mutant background accumulating the $\mathrm{AF}$ intermediate versicolorin A (Skory et al. 1992), similarly showed increased versicolorin A production and increased transcription of an AF and versicolorin A biosynthetic gene, aflC (formerly norA, data not shown).

\section{Induction of ST and AF by exogenous oleic acid.}

We next sought to mimic the $\triangle$ odeA phenotype by addition of oleic acid as free salt or as Tween 80 (a soluble monooleate compound) to standard growth medium. Both compounds induced higher ST accumulation in wild-type A. nidulans (A26) in combination with glucose (data from Tween 80 shown in Figure $2 \mathrm{~A})$. In the presence of glucose, oleic acid $\left(\mathrm{C}_{18: 1}\right)$ significantly increased stc gene transcription (Fig. 2B, stcU) over glucose alone and over glucose with hexanoic acid $\left(\mathrm{C}_{6}\right)$, myristic acid $\left(\mathrm{C}_{14}\right)$, or stearic acid $\left(\mathrm{C}_{18}\right.$, data not shown). Oleic acid (as Tween 80) supplementation of yeast extract sucrose (YES) medium was also found to elicit higher levels of AF and increased transcription of aflC in A. flavus and A. parasiticus (Figs. 2C and D; data from A. flavus). However, addition of oleic acid as a sole carbon source yielded mixed results with generally just a slight increase in ST or AF production compared to that of glucose (data not shown). These data appear to support a synergism between lipids and sugars in ST and AF induction and a putative role for oleic acid induction of $\mathrm{AF}$ and ST gene expression.

\section{Increased peroxisomal activity and concomitant proliferation of peroxisomes in oleate desaturase mutants.}

In addition to what appeared to be induction of AF and ST gene expression and subsequent increased AF and ST biosynthesis by oleic acid, we also considered it possible that increased toxin formation could be associated with increased peroxisomal 
fatty acid $\beta$-oxidation. Oleic acid is a known peroxisome proliferator in A. nidulans and other fungi, a phenomenon associated with increased $\beta$-oxidation (Valenciano et al. 1996; Veenhuis et al. 1987). Examination of the A. nidulans $\Delta$ odeA mutant grown on glucose minimal medium showed that, in addition to demonstrating increased intracellular levels of oleic acid, it also exhibited canonical signs of increased peroxisomal activity, including peroxisomal proliferation. For example, transcription of two genes encoding components of the $\beta$-oxidation pathway, the D-bifunctional protein homolog (foxA, Maggio-Hall and Keller 2004) and a putative peroxisomal thiolase gene ( $3 \mathrm{kt})$, was up-regulated in the $\triangle o d e A$ strain grown on glucose medium compared with that in wild type (Fig. 3A). Additionally, wholecell catalase activity, a marker for peroxisomal $\beta$-oxidation (Veenhuis et al. 1987), was significantly higher in $\triangle$ odeA as compared with wild type (Fig. 3B). Finally, whereas the peroxisomes of wild type grown on $1 \%$ glucose minimal medium (GMM) are uniformly distributed throughout the hyphae, the clustering of peroxisomes (visualized by an isocitrate lyasegreen fluorescent protein (GFP) fusion) in GMM-grown $\triangle$ odeA hyphae more closely resembled that of wild type grown in oleic acid, thus visually supporting a case for peroxisomal proliferation in this mutant (Fig. 3C).

\section{Role for peroxisomal $\beta$-oxidation in $\mathrm{ST}$ biosynthesis.}

The increased fox $A$ and $3 k t$ gene expression suggested a role for peroxisomal $\beta$-oxidation in providing acetyl-CoA moeities for AF or ST production. Therefore, stc gene expression and $\mathrm{ST}$ production were analyzed in a $\triangle$ foxA single mutant and a $\triangle o d e A-\triangle$ foxA double mutant to determine any dependence on $\beta$-oxidation for ST production. Disruption of foxA (RLMH12), leading to abolishment of peroxisomal $\beta$-oxidation (MaggioHall and Keller 2004), affected ST production in a mediumdependent manner. No effect on ST production was evident on lactose minimal media (Fig. 4B) or GMM (Fig. 1A), but considerably less ST production was seen on glucose plus Tween 80 (Fig. 4B). Interestingly, transcription of stc genes was higher in $\triangle f o x A$ than in wild type, and the transcripts persisted longer (Fig. 4A).

On GMM the double mutant ( $\triangle o d e A ; \Delta f o x A$, RLMH14) generated slightly less ST than the $\Delta$ odeA single mutant (Fig. 1A). However, stc gene transcription was increased and commenced earlier than in wild type or the $\Delta o d e A$ mutant alone (Fig. 1B). These results support a role for the accumulated fatty acids, particularly oleic acid, in both induction of transcription of stc genes and in providing carbon via $\beta$-oxidation to the ST backbone.

\section{Mitochondrial $\beta$-oxidation is required for $\mathrm{ST}$ production in oatmeal medium and on live corn kernels.}

We have recently demonstrated that $A$. nidulans also has a mitochondrial $\beta$-oxidation pathway (Maggio-Hall and Keller 2004). Mutation of this pathway produced a more severe growth phenotype on most fatty acids $\left(\mathrm{C}_{4}\right.$ to $\left.\mathrm{C}_{18}\right)$ than mutation of the peroxisomal pathway. Therefore, to examine a possible contribution of mitochondrial $\beta$-oxidation to toxin formation, strains carrying a disruption of the mitochondrial enoylCoA hydratase $(\Delta e c h A)$ were analyzed for ST production. The contributions of mitochondrial $\beta$-oxidation appeared minimal in sugar or sugar and lipid based media. For example, no effect was observed on lactose minimal medium (Fig. 4B). ST production in the presence of glucose and Tween 80 was less than that of the wild-type strain (Fig. 4B), but the effect was less severe than that observed for the peroxisomal $(\triangle$ foxA $)$ mutant. The $\Delta e c h A ; \Delta f o x A$ double mutant also produced wild-type levels of ST on lactose but produced nearly undetectable levels of ST on glucose with Tween 80 (Fig. 4B).

Plant seeds are the agricultural commodities most affected by AF contamination. Seeds obviously represent a complex carbon source, containing not only lipids but also starch, sugars, and proteins (Watson 1987). In order to assess the contribution of fatty acid metabolism to mycotoxin production in the presence of these other potential carbon sources, $A$. nidulans $\beta$ oxidation mutants $(\triangle$ fox $A, \triangle e c h A$, and $\triangle e c h A ; \triangle$ fox $A)$ were grown on both oatmeal medium and live corn kernels. Although ST production was near normal in the $\Delta$ foxA strain, only trace ST accumulation was observed on solid oatmeal medium for strains carrying a mutation in mitochondrial $\beta$-oxidation $(\triangle e c h A$, data not shown). Since a small growth defect was observed on this medium for $\Delta e c h A$ strains (Fig. 5A), ST was extracted from equal weights $(0.05 \mathrm{~g}$ dry weight $)$ of mycelium grown in liquid oatmeal medium. Again, the $\Delta e c h A$-carrying strains did not accumulate visible levels of ST (Fig. 5B). Efforts to obtain quality RNA from oatmeal-grown $A$. nidulans strains were unsuccessful, so it is unknown if stc genes were expressed in the $\Delta e c h A$ strains under these conditions. The same $A$. nidulans strains were also inoculated onto wounded, surface-sterilized living corn kernels. While growth of all strains was ob-

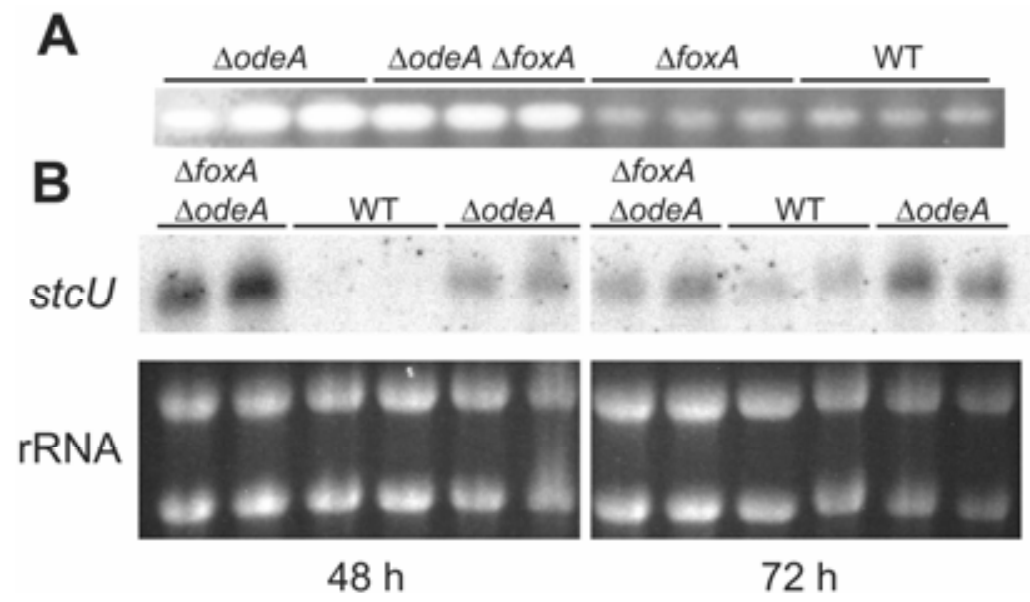

Fig. 1. Increased stc gene expression and sterigmatocystin (ST) synthesis by an Aspergillus nidulans oleate desaturase mutant, $\Delta$ odeA. A, Thin-layer chromatography analysis of ST accumulation in TAMC31.65 ( $\Delta$ odeA), RLMH14 ( $\Delta$ odeA, $\Delta$ foxA), RLMH12 ( $\Delta$ foxA), and A26 (wild type, WT). Each strain is shown in triplicate (from three independent cultures). ST was extracted from solid $1 \%$ glucose minimal medium (GMM) after 72 h of incubation. B, Northern analysis of $s t c U$ expression in GMM-grown strains at 48 (left panel) and $72 \mathrm{~h}$ (right panel). Shown are RLMH14 ( $\Delta$ odeA, $\Delta$ foxA), A26 (WT), and TAMC31.65 ( $\triangle$ odeA). Ethidium bromide-stained rRNA bands are shown as a control for total RNA loading. 
served (as assessed by the appearance of green conidia at the wound site; Fig. 6A), strains carrying $\Delta e c h A$ produced markedly less ST than did wild type (Fig. 6B). The double mutant, $\triangle$ fox $A ; \Delta e c h A$, generated only trace amounts of ST. The conidia produced on the corn kernels were tested to verify the phenotype of the inoculated strain, fulfilling Koch's postulate (data not shown).

\section{The AF or ST precursor norsolorinic acid accumulates in the peroxisome.}

Considering the essential contribution of $\beta$-oxidation to ST production in planta and its important contributions in culture media, we thought it possible that the early, acetyl-CoA-requiring steps of ST and AF synthesis would be located in close proximity to $\beta$-oxidation pathways, possibly even in the peroxisomes or mitochondria. Part of the penicillin biosynthetic pathway takes place in the peroxisome, thus establishing the precedent of this organelle's importance in secondary metabolism (Muller et al. 1991).

To determine the location of the first steps of ST and AF synthesis, we investigated the location of norsolorinic acid

A

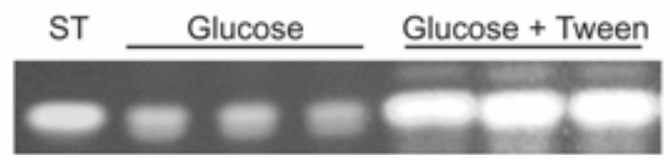

B

C

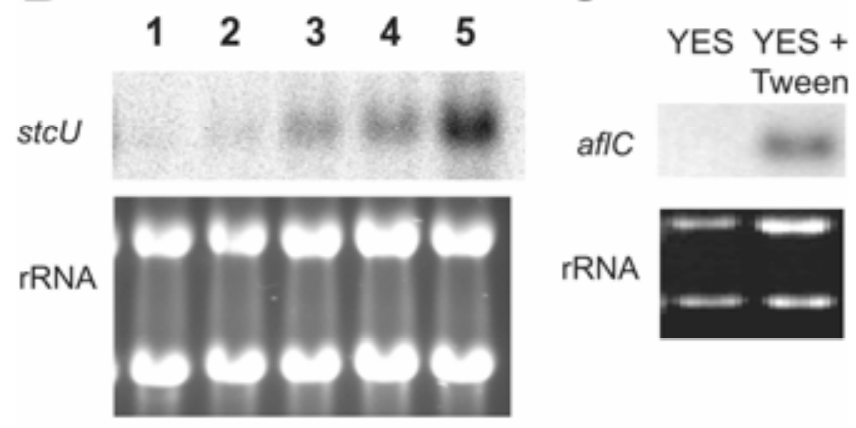

D

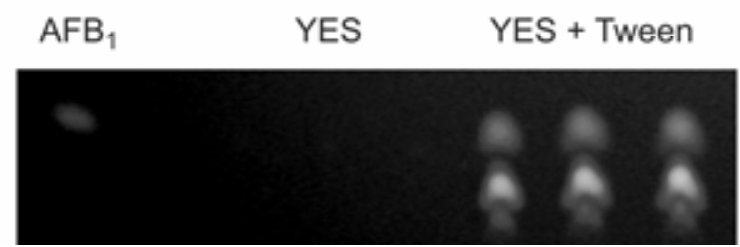

Fig. 2. Increased sterigmatocystin (ST) or aflatoxin (AF) gene expression and ST and AF synthesis by addition of exogenous oleic acid. A, Thinlayer chromatography (TLC) analysis of ST accumulation by Aspergillus nidulans A26 (WT) grown on solid 1\% glucose minimal medium (GMM) with and without Tween $80(1 \%)$. Cultures were incubated 72 h. B, Northern analysis of $s t c U$ gene expression in wild-type A. nidulans (A26) grown on GMM plus $20 \mathrm{mM}$ hexanoic acid (lane 1), $7.5 \mathrm{mM}$ myristic acid (lane 2), no addition (lane 3), tergitol only (lane 4), and $6 \mathrm{mM}$ oleic acid (lane 5); $1 \%$ tergitol NP40 was present in all cultures except that represented in lane 3. C, Northern analysis of aflC (norA) expression in wild-type A. flavus (12S) grown on yeast extract sucrose (YES) medium with and without $1 \%$ Tween 80 . Total RNA was obtained after $72 \mathrm{~h}$ of incubation. Ethidium bromide-stained rRNA is shown as a control for total RNA loading. D, TLC analysis of AF accumulation by A. flavus (12S) grown on YES medium with and without $1 \%$ Tween 80 . The lower spot in YES + Tween lanes is AF G1. AF was extracted from liquid shake cultures after $72 \mathrm{~h}$ of incubation.

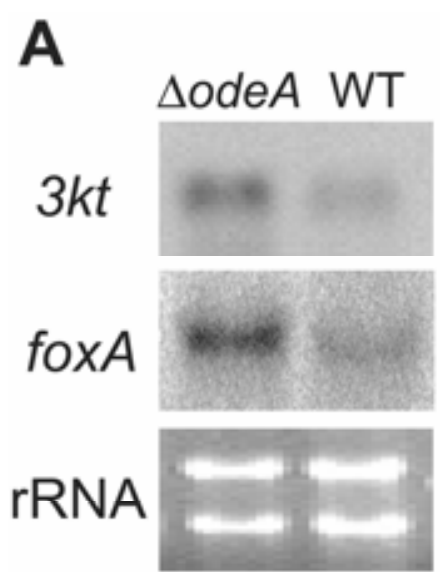

B
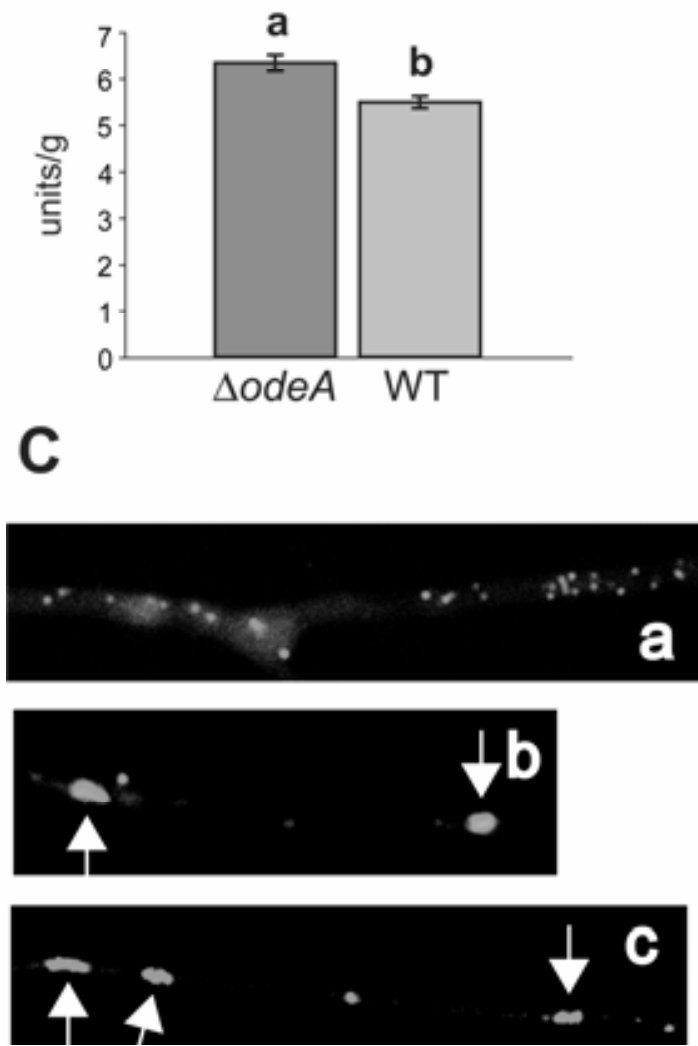

Fig. 3. Evidence of peroxisome proliferation and increased peroxisomal $\beta$ oxidation in the Aspergillus nidulans oleate desaturase mutant. A, Northern analysis of $\beta$-oxidation gene expression in $1 \%$ glucose minimal medium (GMM)-grown TAMC31.65 ( $\triangle$ odeA) and A26 (WT). Shown are a putative peroxisomal thiolase $(3 k t)$ and $f o x A$, encoding the bifunctional enoyl-CoA hydratase/hydroxyacyl-CoA dehydrogenase. Ethidium bromide-stained rRNA is shown as a control for total RNA loading. B, Enzymatic measure of catalase activity from cell-free extracts. Shown are results from TAMC31.65 ( $\triangle$ odeA) and A26 (WT). Specific activity measurements are statistically different, as assessed by SAS analysis of variance (indicated by a and b). A unit is defined as the amount of enzyme that liberates half the peroxide oxygen from a hydrogen peroxide solution of any concentration in $100 \mathrm{~s}$ at $25^{\circ} \mathrm{C}$. C, Fluorescence micrographs showing peroxisome proliferation in the glucose-grown $\triangle o d e A$ strain. Peroxisomes fluoresce green due to the introduction of an isocitrate lyase-green fluorescent protein fusion gene (Maggio-Hall and Keller 2004; Weeradechapon 2002) but appear white after conversion of the micrograph to grayscale. Panels a and b show odeA wild-type RRAW14.2 (acuD::gfp) grown on GMM and GMM plus Tween 80, respectively. Panel c shows RRAW10.4 ( $\triangle$ odeA; $a c u D:: g f p$ ) grown on GMM. Examples of peroxisome clustering are indicated with arrows. 
(NOR) in the fungal cell. NOR, a fluorescent compound and the first stable intermediate of ST and AF biosynthesis, is produced by the activity of the ST and AF polyketide synthase (PKS) and fatty acid synthase (FAS), which are believed to form a complex (Watanabe and Townsend 2002). The PKS/FAS complex forms the acetyl-CoA-derived backbone of the ST and AF molecules, an unstable intermediate called noranthrone that is hypothesized to be immediately converted to NOR by an unknown, possibly nonenzymatic mechanism (Watanabe and Townsend 2002). In NOR-accumulating mutants of A. nidulans, red-fluorescent NOR was observed to transiently localize to specific hyphal organelles in Tweengrown cultures, accumulating to visible levels by $48 \mathrm{~h}$ and dispersing by $72 \mathrm{~h}$. Introduction of the isocitrate lyase-GFP fu- sion construct into the NOR strain (RRAW14.2) enabled us to identify these organelles as peroxisomes (Fig. 7A and B). NOR was also observed in organelles of NOR-accumulating mutants of $A$. flavus (MAEW4) and A. parasiticus (SRRC126). These organelles appeared to be peroxisomes, based on their aggregation behavior in the presence of fatty acids (Fig. 7E and F, A. parasiticus shown).

\section{DISCUSSION}

Despite advances in identifying the genes responsible for toxin biosynthesis in many fungal plant pathogens, there has been little progress in understanding the regulation of toxin biosynthesis, particularly during the host-pathogen interaction.

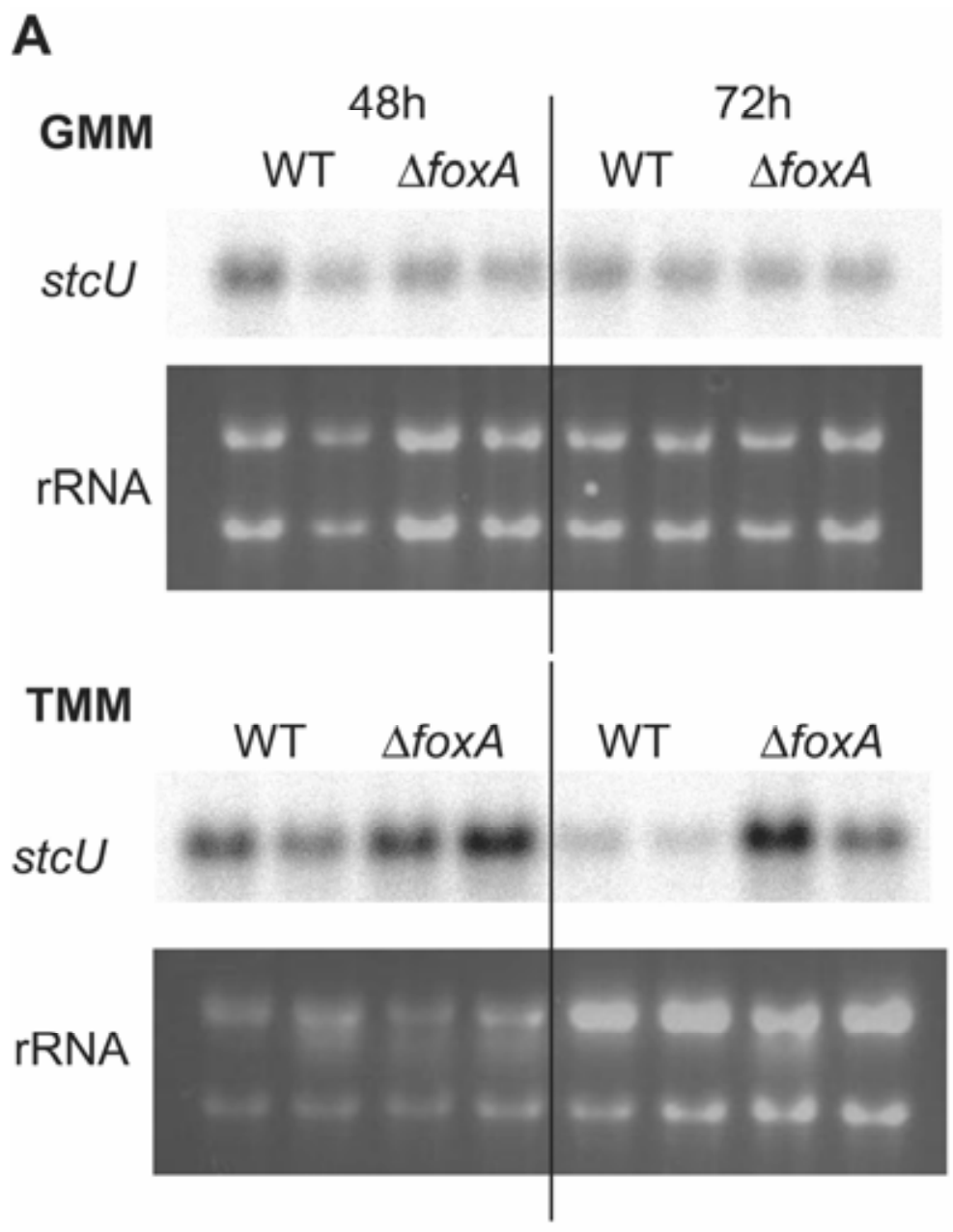

B

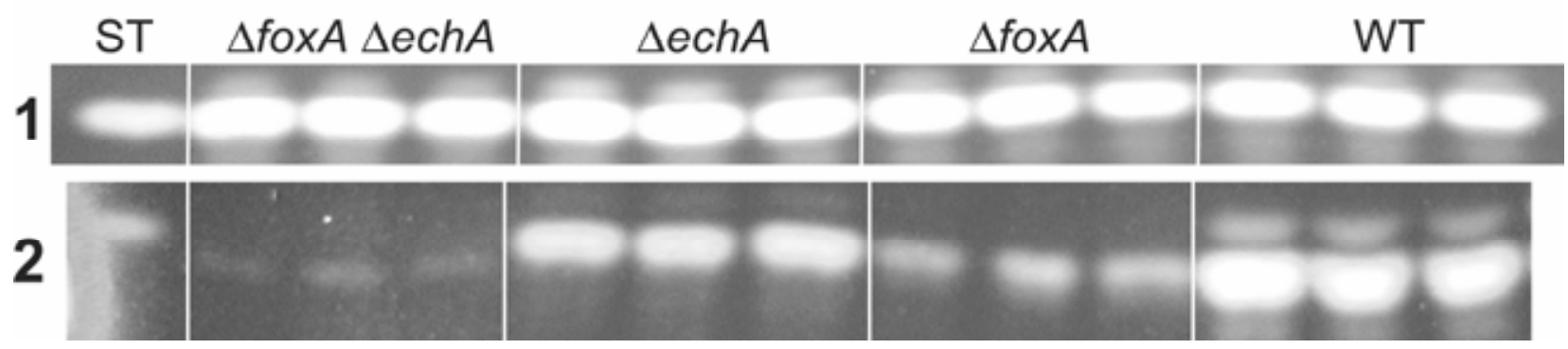

Fig. 4. Role of peroxisomal $\beta$-oxidation in sterigmatocystin (ST) accumulation and gene expression. A, Northern analysis of $s t c U$ expression in A26 (WT) and RLMH12 ( $\triangle$ foxA). Shown are results from 1\% glucose minimal medium (GMM) (top panel) and Tween 80 minimal medium (TMM) (bottom panel) at both 48 and $72 \mathrm{~h}$. Both GMM and TMM were inoculated with mycelium grown $24 \mathrm{~h}$ in GMM. Ethidium bromide-stained rRNA is shown as a control for total RNA loading. B, Thin-layer chromatography analysis of ST accumulation in strains grown on lactose minimal medium (1) and GMM plus $1 \%$ Tween 80 (2). Shown are TLMH7 ( $\triangle$ foxA $\triangle e c h A)$, RLMH41 ( $\Delta e c h A)$, RLMH12 ( $\Delta$ foxA), and A26 (WT). Solid media were extracted after $72 \mathrm{~h}$ of incubation. 
Whereas a wealth of studies have shown that pathogen developmental processes, i.e., appressorium formation, are instigated in response to plant signals - be they structural or chemical in nature (Dickman et al. 2003; Hoch et al. 1987; Howard and Valent 1996; Hwang et al. 1995), there has been a paucity of studies investigating host contributions towards toxin produc- tion in fungi. This lack of knowledge is especially notable in fungal-seed interactions, which are infamous for production of secondary metabolites known as mycotoxins. Here, we present several lines of evidence implicating the importance of plant fatty acids in contributing to production of the polyketides AF and ST in Aspergillus spp. Genetic and biochemical investiga-
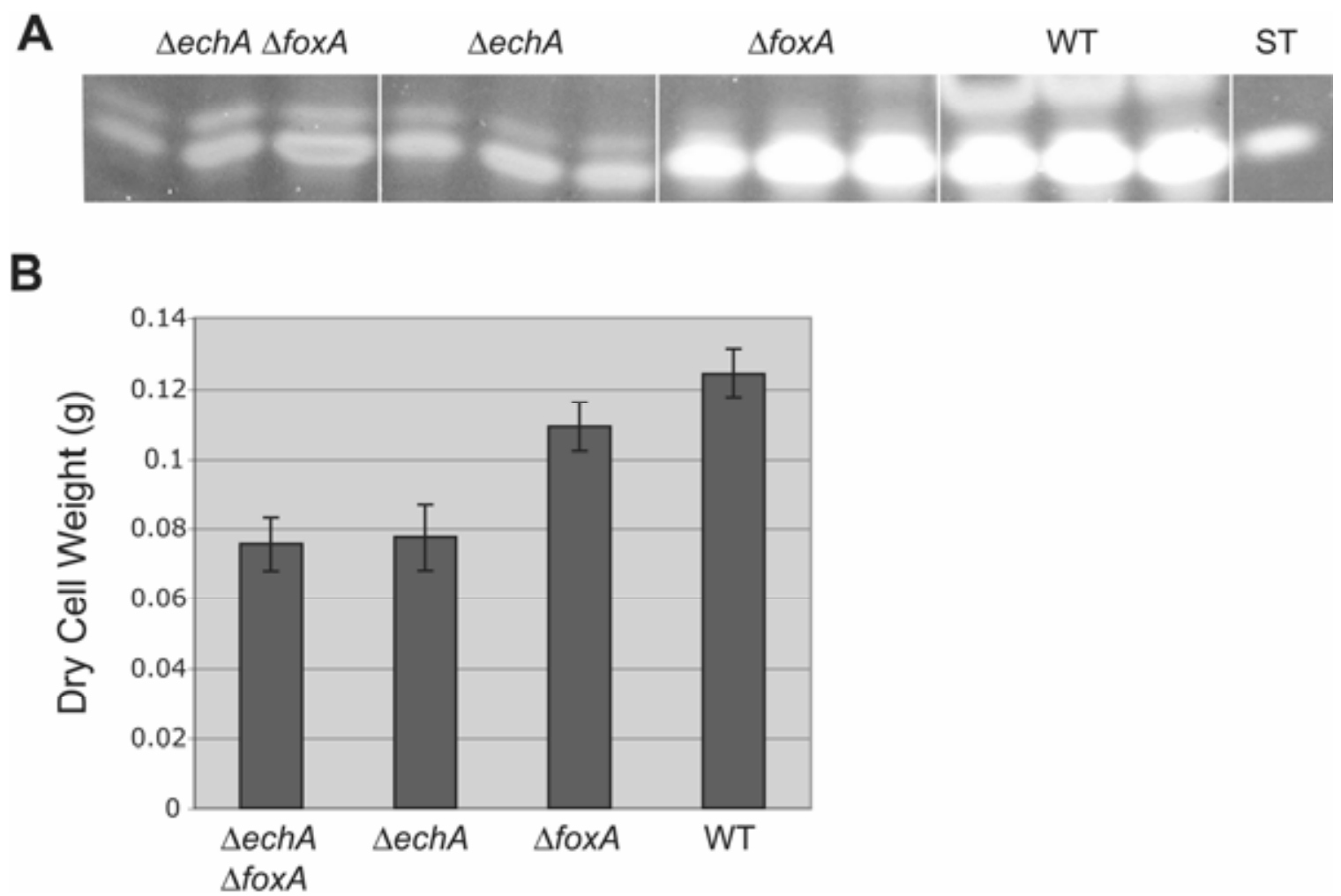

Fig. 5. Mitochondrial $\beta$-oxidation is essential for sterigmatocystin (ST) synthesis on oatmeal medium. A, Thin-layer chromatography analysis of ST

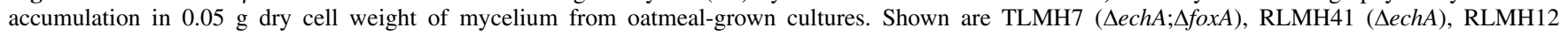
$(\Delta f o x A)$, and A26 (WT). Cultures were grown $72 \mathrm{~h}$. Fluorescent spots in $\Delta e c h A ; \Delta f o x A$ and $\Delta e c h A$ lanes are not ST and run slightly below and above ST. B, Yield of mycelium per 50-ml culture.

A

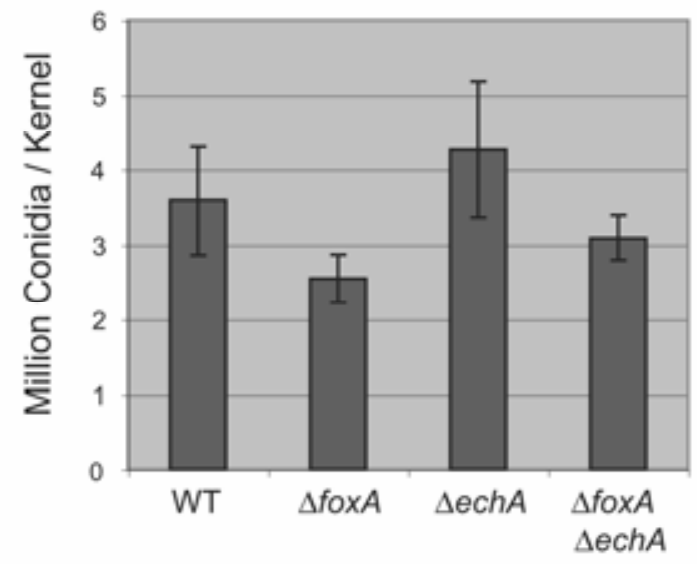

B

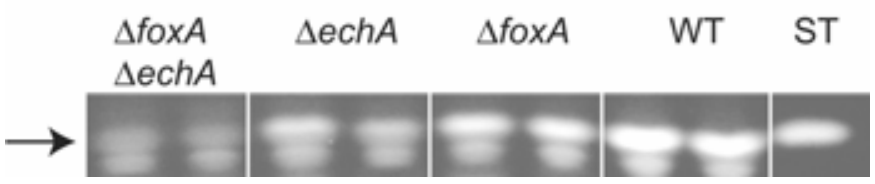

Fig. 6. $\beta$-oxidation is required for optimal sterigmatocystin (ST) synthesis on corn kernels. A, Yield of conidia (average from five kernels) after inoculation with A26 (WT), RLMH12 $(\Delta f o x A)$, RLMH41 $(\Delta e c h A)$, and TLMH7 $(\Delta f o x A ; \Delta e c h A)$. Error bars represent standard error. B, Thin-layer chromatography analysis of ST extracted from Aspergillus nidulans-infected corn kernels. Strains are as indicated for A. The band corresponding to ST is indicated by the arrow. 
tions of the model organism A. nidulans and the field pathogens $A$. flavus and $A$. parasticus indicate that fatty acids, particularly oleic acid, are important in inducing ST and AF gene expression in planta and, furthermore, that these same fatty acids are then utilized via $\beta$-oxidation processing in the Aspergilli as a carbon source for the ST and AF polyketide chain. Results from this study also highlight the differences in ST and AF regulation in artificial growth media versus in planta and provide a cautionary tale in over interpreting results of toxin production from laboratory experiments, as such results may not reflect toxin production in the field.
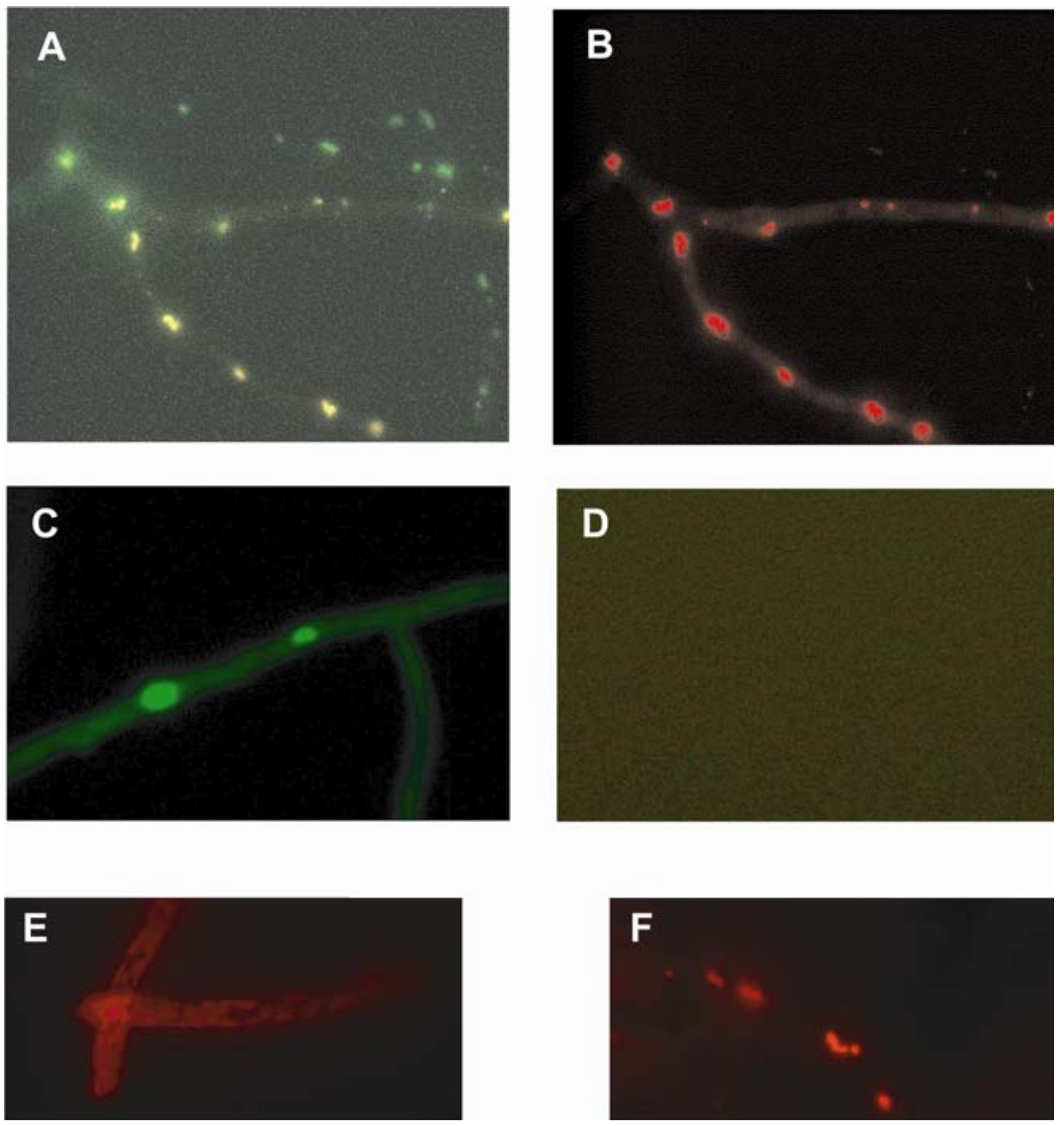

Fig. 7. Peroxisomal accumulation of norsolorinic acid (NOR). NOR was visualized using epifluorescence microscopy (tetramethylrhodamine isothiocyanate [TRITC] filter). An Aspergillus nidulans strain carrying an isocitrate lyase- green fluorescent protein fusion was used to enable visualization of peroxisomes. A and B, The same mycelium (RRAW 14.2: $a c u D:: g f p, \Delta s t c E:: \arg B$ ) under fluorescein isothiocyanate (FITC) (green) and TRITC (red) filters, respectively. C and D, Mycelium from a non-NOR-producing control strain (RRAW10.5: acuD::gfp) under FITC and TRITC filters, respectively. E and F, A. parasiticus SRRC126 grown on yeast extract sucrose (YES) and YES plus Tween 80, respectively. Mycelium is shown under the TRITC filter to visualize NOR in compartments that are consistent with peroxisomes, based on aggregation behavior in the presence of Tween $80(\mathrm{~F})$. 
for greatest expression of these genes in growth media. The molecular mechanism for this phenomenon remains to be identified and may differ from fatty acid-dependent regulation of fatty acid anabolism genes, which involves a conserved feedback mechanism aimed at maintaining optimum saturated to unsaturated fatty acid ratios in the fungus (Calvo et al. 2001; Wilson et al. 2004; Xu et al. 1999). Additionally, short-chain fatty acids such as hexanoic acid induce expression of fatty acid catabolism genes (Maggio-Hall and Keller 2004) but not ST and AF biosynthetic genes (this study). Our finding that saturated fatty acids (myristic and stearic) failed to induce stc gene expression could indicate that Aspergillus ST and AF genes have evolved to respond directly to unsaturated fatty acids, which make up the majority of seed fatty acids.

\section{Oleic acid as a peroxisomal activator stimulates toxin formation.}

High ST and AF gene expression and subsequent product formation was particularly noticeable in $\triangle o d e A$ mutants and by oleic acid supplementation of wild-type strains. Whereas part of this increase is likely associated with the ST and AF gene-inducing properties of oleic acid, its derivatives, or both, our data also implicate an additional role for oleic acid associated with increased peroxisomal activity. Studies in several organisms, including $A$. nidulans, have established oleic acid as a peroxisomal proliferation agent (Tanaka et al. 1982; Valenciano et al. 1996, 1998; Veenhuis et al. 1987). Concomitant with proliferation is a significant increase in catalase and $\beta$-oxidation activity.

Similar to other studies (Valenciano et al. 1996, 1998), we observed physical and biochemical signs of increased peroxisomal activity in oleic acid-fed cultures of Aspergillus spp. Strikingly, these signs were also observed in the glucosegrown $\triangle$ odeA strain. Most significantly, the expression of two peroxisomal $\beta$-oxidation genes was higher in this mutant than in wild type (Fig. 3). As $\beta$-oxidation is one of the prime suppliers of cellular pools of acetyl-CoA, the increased foxA and $3 k t$ expression suggested oleic acid might also be contributing to ST and AF biosynthesis by virtue of increased $\beta$-oxidation of exogenous fatty acids leading to increased acetyl-CoA units. Such a hypothesis would lead to the prediction that oilseed containing high levels of oleic acid would stimulate the greatest amount of ST and AF production. Indeed, this is exactly what has been recently demonstrated in studies of AF contamination of high-oleic versus low-oleic peanut lines (Xue et al. 2003).

This hypothesis was also supported by examination of an $A$. nidulans double mutant $(\triangle$ fox $A ; \triangle$ ode $A)$ that had increased oleic acid accumulation but was blocked in peroxisomal $\beta$-oxidation. Transcription of stc genes was increased in the double mutant compared with that in $\triangle o d e A$ alone; however, the yield of ST was actually less. This result highlights the two roles fatty acids may play in ST and AF production, the first being that of an inducer of gene expression and the second being a source of acetyl-CoA as substrate for ST and AF biosynthesis.

\section{Requirement of peroxisomal and mitochondrial $\beta$-oxidation in toxin production differs in planta versus in defined media.}

When fatty acid $\beta$-oxidation was reduced in A. nidulans through deletion of either foxA or echA, no to little effect on ST production was observed in the mutants grown on glucose or lactose as a sole carbon source. This suggested that any lipids stored by the fungus grown on simple sugars do not represent a major source of carbon for synthesis of ST and AF, and we speculate that acetyl-CoA units derived from glycolysis present the major source of carbon for the polyketides in this situation. However, exogenous sources of lipids do provide a substantial-even preferred-source of carbon in mixed media (sugar plus lipids) and are essential when the fungus is grown on complex plant materials or living seed as demonstrated by the decrease or complete loss of ST production in $\triangle$ foxA or $\Delta e c h A$ mutants, or both, on these substrates.

Interestingly, the relative contributions of the two $\beta$-oxidation pathways to ST production differed dependent on substrate. Disruption of mitochondrial $\beta$-oxidation had a larger effect on ST production on oatmeal than disruption of the peroxisomal pathway, whereas both pathways appeared to contribute at similar levels on corn. A larger dependence on peroxisomal $\beta$ oxidation was observed on laboratory media containing oleic acid or Tween 80 . Phenotypically, the echA-dependent pathway is required for growth on fatty acids of any length, while the foxA-dependent pathway is only required for growth on very long-chain fatty acids (Maggio-Hall and Keller 2004). The relative contribution of either pathway to ST synthesis likely depends on the specific fatty acid composition of the host seed. While $\mathrm{C}_{18}$ fatty acids (stearic, oleic, linoleic, and linolenic) make up the majority of corn fatty acid content, significant amounts of palmitic acid $\left(\mathrm{C}_{16}\right)$ are also present (ranging from 6 to $22 \%$ of total) (Jellum 1970). It is also possible that many shorter chain fatty acids are released during pathogen degradation of plant cells.

\section{Cellular localization of st/af biosynthesis.}

Finding of the ST and AF PKS-FAS product NOR in the peroxisomes raises the question whether NOR is synthesized in or transported to the peroxisome. Peroxisomes house the final step in fungal penicillin biosynthesis (Muller et al. 1991), establishing a precedent for this organelle's involvement in secondary metabolism. However, a peroxisomal localization of a PKS would be unprecedented, and indeed, analysis of the amino acid sequence by various sorting algorithms does not predict this (data not shown). Algorithms also do not predict a peroxisomal location for the ST and AF FAS; however, proteins lacking either of the two recognized peroxisome-targeting signal sequences may still be successfully transported to this compartment (Klein et al. 2002).

The ST and AF FAS first generates hexanoyl-CoA from acetyl-CoA and malonyl-CoA. However, studies have shown that ST production can be remediated in ST FAS mutants when grown on hexanoate-supplemented medium (Brown et al. 1996a; Zhang and Keller 2004), raising the possibility that seed short-chain fatty acids could contribute starter units. Hexanoyl-CoA is the starter unit and malonyl-CoA the extender units used by the ST and AF PKS to generate noranthrone, the immediate precursor to NOR. The ST and AF PKS and FAS are believed to interact physically, thus suggesting a common subcellular localization (Watanabe and Townsend 2002).

Are the ST and AF PKS and FAS substrates available within the peroxisome? Although acetyl-CoA will be produced in the peroxisome, the mitochondria, or both during $\beta$-oxidation, there are no data supporting or refuting malonyl-CoA production or transport in these organelles in fungi. The conversion of acetyl-CoA to malonyl-CoA by acetyl-CoA carboxylase (ACC) is only reported to occur in the cytoplasm in yeast and mammals (Hasslacher et al. 1993; López-Casillas et al. 1988). Consistent with yeast and mammals, Aspergilli appear to contain only one ACC (Morrice et al. 1998). Plants contain an extra ACC in their plastids (Choi et al. 1995), but examination of the Aspergillus genomes does not show any obvious homolog to subunits of this enzyme. Although we have not identified any studies examining possible transport of malonyl-CoA into peroxisomes, malonyl-CoA production in mammalian perox- 
isomes during degradation of odd-chain dicarboxylic acids has been reported, and malonyl-CoA decarboxylase is required to prevent toxic accumulation of malonyl-CoA from this process (Sacksteder et al. 1999). This enzyme is not found in fungi, leaving open, for the moment, how or if malonyl-CoA is present in fungal peroxisomes. Exclusion of malonyl-CoA from the peroxisome would rule out NOR synthesis in this organelle.

Attempts to visualize StcA (ST PKS) within hyphae were unsuccessful in our lab. However, we observed that a GFP fusion to StcJ, the FAS $\alpha$-subunit for the ST pathway in $A$. nidulans (Brown et al. 1996a), localized to a nonperoxisomal organelle (L. A. Maggio-Hall and N. P. Keller, unpublished data). Nonetheless, due to the conditions employed (i.e., overexpression of a recombinant fusion protein), the physiological relevance of the observed localization remains to be determined. NOR ketoreductase, catalyzing the next downstream step after NOR synthesis in the ST and AF pathway, has been observed in the cytoplasm of A. parasiticus (Lee et al. 2004).

In conclusion, based on the results reported here, we present a mechanistic model emphasizing the critical importance of seed fatty acids and fungal $\beta$-oxidation in generating the mycotoxins ST and AF in the Aspergillus-seed interaction. This relationship involves both transcriptional regulation of ST and AF gene expression and acyl-CoA provision for the ST and AF polyketide enzymes. Our model postulates that other fatty acid metabolism proteins - possibly both seed and fungal in originwould also be required for ST and AF production in planta. These include triglyceride lipases, already implicated in pathogenicity (Eddine et al. 2001; Smart et al. 1990; Yu et al. 2003), and fatty acid activation and transport proteins. We are also interested in determining if these findings represent a paradigm for generation of fungal polyketides implicated in other plant-microbe interactions. Although not mechanistically examined, the production of the fungal polyketides lovastatin and geodin was positively correlated with increased transcription of fatty acid metabolism genes, including $\beta$-oxidation genes, in A. terreus (Askenazi et al. 2003).

\section{MATERIALS AND METHODS}

\section{Fungal strains and growth conditions.}

A. nidulans, A. flavus, and A. parasiticus strains used in this study are listed in Table 1. A. nidulans was grown on GMM or $1 \%$ lactose minimal medium (Calvo et al. 2001) with the appropriate supplements for auxotrophic markers (Kaefer 1977). Polysorbate monooleate (Tween $80,1 \% \mathrm{vol} / \mathrm{vol}$ ) or oleic acid (6 mM) in 1\% (vol/vol) Tergitol NP40, was added to GMM or substituted for glucose completely. The oleate portion of Tween 80 has been shown to be released and incorporated as oleic acid into cells of A. niger (Nemec and Jernejc 2002). Solid medium was obtained by the addition of agar (16 g per liter). A. nidulans cultures were grown in the dark at $37^{\circ} \mathrm{C}$. Cultures of $A$. parasiticus and A. flavus were grown on YES medium (2\% yeast extract, $20 \%$ sucrose) or YES supplemented with $1 \%$ Tween $80(\mathrm{vol} / \mathrm{vol})$ at $29^{\circ} \mathrm{C}$ in the light.

\section{Mycotoxin extraction and visualization.}

ST extraction and visualization from minimal media. Spores $\left(10^{6}\right)$ of the appropriate $A$. nidulans strains were added to $5 \mathrm{ml}$ of molten but cool GMM. This was then poured onto 25-ml plates of solid GMM, which were allowed to set and were incubated for $72 \mathrm{~h}$. After this time, $3 \times 12-\mathrm{mm}$ cores were punched from each plate and were homogenized in $3 \mathrm{ml}$ of sterile water. Chloroform $(3 \mathrm{ml})$ was added, and the samples were vortexed vigorously and were centrifuged for $15 \mathrm{~min}$ at $1,500 \times g$ ). The bottom layer was transferred to a small glass vial and was allowed to evaporate overnight. The sample was then resuspended in $100 \mu \mathrm{l}$ of chloroform, and $25 \mu \mathrm{l}$ was applied to silica thinlayer chromatography (TLC) plates (Whatman, Florham Park, NJ, U.S.A.). TLC developing and ST detection procedures have been previously described (Brown et al. 1996a).

ST extraction from oatmeal medium. Spores $\left(10^{6}\right)$ were inoculated into $50 \mathrm{ml}$ of liquid oatmeal medium ( $1 \%$ homogenized Quaker old-fashioned oats). Flasks were shaken at $300 \mathrm{rpm}$ at $37^{\circ} \mathrm{C}$ for $72 \mathrm{~h}$. Mycelium was harvested by filtration through Miracloth (Calbiochem, EMD Biosciences, San Diego, CA, U.S.A.) and was lyophilized overnight. Mycelium was weighed and $0.05 \mathrm{~g}$ of each sample was resuspended in $3 \mathrm{ml}$ of $\mathrm{ddH}_{2} \mathrm{O}$ and extracted with chloroform, as described above. TLC conditions were identical to those used for samples from minimal medium (discussed above).

ST extraction from corn. Corn kernels were wounded with a dissecting probe at the embryo, were surface-sterilized with $10 \%$ bleach ( $1 \mathrm{~min}$.), and were washed with sterile $\mathrm{ddH}_{2} \mathrm{O}(1$ min). Sterile kernels were transferred to humid chambers (sterile glass petri dishes with two layers of wetted Whatman paper and a small water reservoir). Each kernel was inoculated at the wound site with 5,000 spores (in $5 \mu \mathrm{l}$ of water, 35 kernels per strain). Petri dishes were wrapped in aluminum foil and were stored at $29^{\circ} \mathrm{C}$ for one week. Approximately $1 \mathrm{ml}$ of sterile $\mathrm{ddH}_{2} \mathrm{O}$ was added to the filter paper every other day to maintain humidity without inducing kernel germination. Five kernels were saved for spore counting. ST was extracted from 15 kernels as follows. Kernels were ground with a mortar and pestle with $5 \mathrm{ml}$ of acetone. After addition of $10 \mathrm{ml}$ of an acetone/water solution (7:3), the cornmeal was stirred and the liquid was squeezed out (using the mortar) and transferred to a $50-\mathrm{ml}$ Falcon tube. After addition of $10 \mathrm{ml}$ of hexane, the tubes were vortexed and centrifuged $(10 \mathrm{~min}$. at $1,500 \times \mathrm{g})$. The bottom (aqueous) layer was transferred to a new Falcon tube and was allowed to dry overnight in a flow hood. The residue was resuspended in $10 \mathrm{ml}$ of $\mathrm{ddH}_{2} \mathrm{O}$ and was extracted with an equal volume of chloroform. After vortexing and centrifuging, the bottom (organic) layer was transferred to a new Falcon tube and was allowed to evaporate. The residue was resuspended in $10 \mathrm{ml}$ of $\mathrm{ddH}_{2} \mathrm{O}$ and was extracted with an equal volume of hexane. The hexane layer was evaporated for TLC analysis (described above), and the aqueous layer was extracted with chloroform. The bottom (organic layer) was evaporated for TLC analysis (described above).

$A F$ extraction. A 50-ml concentration of liquid YES and YES $+1 \%$ Tween $80(\mathrm{vol} / \mathrm{vol})$ media was inoculated with $10^{6}$ spores of appropriate strains. Cultures were shaken (300 rpm) at $29^{\circ} \mathrm{C}$ for $72 \mathrm{~h}$, under constant light conditions. To each culture was added an equal volume of acetone, followed by an equal volume of chloroform. After separation, the chloroform layer was transferred to a glass vial and was allowed to evaporate. The sample was then resuspended in $500 \mu \mathrm{l}$ of chloroform, and $1 \mu \mathrm{l}$ was spotted onto glass-backed TLC plates. TLC developing and visualization of AF were identical to that used for ST, as previously described (Brown et al. 1996a).

Quantification of ST by LC/MS. LC/MS quantification of ST was achieved using a Thermo-Finnigan LCQ deca quadrupole ion trap. The effluent from autosampler (TSP AS3000) and pump (T4000) was coupled directly to the MS via the atmospheric pressure interface operated in the electrospray (ESI) mode. The column was a 5-micron Intersil ODS3, 15 $\mathrm{cm} \times 0.3 \mathrm{~mm}$ (Metachem Technologies, Torrance, CA, U.S.A.). The flow rate was held constant at $0.3 \mathrm{ml} / \mathrm{min}$. The effluent was transferred into the interface with a spray voltage of $4.5 \mathrm{kV}$. The capillary temperature was $255^{\circ} \mathrm{C}$. The MS was scanned from $\mathrm{m} / \mathrm{z} 250$ to 400 . After the sample $(10 \mu \mathrm{l}$ of a $100-\mu 1$ chloroform extraction) was injected, the solvent was programmed from 65:35:0.35 (water/methanol/acetic acid) to 
5:95:0.35 (water/methanol/acetic acid) over a period of 10 min and was held at the final composition until $25 \mathrm{~min}$. The column was returned to the original solvent composition over a period of $1 \mathrm{~min}$ and was held for $10 \mathrm{~min}$ to reequilibrate before the next sample was injected. Under these conditions ST eluted at approximately $17.5 \mathrm{~min}$. The protonated molecular ion was observed at $\mathrm{m} / \mathrm{z} 325$. Quantification was by the external standard method and was based on the abundance of this signal.

\section{mRNA analysis.}

Total RNA was extracted from lyophilized mycelia using Trizol reagent (Invitrogen, Carlsbad, CA, U.S.A.). RNA (10 to $30 \mu \mathrm{g}$ ) was separated on a $1.3 \%$ agarose and $1.5 \%$ formaldehyde gel. RNA was transferred to Hybond membrane (Amersham Biosciences, Piscataway, NJ, U.S.A.) by capillary action.

RNA transcript analysis was performed using randomprimer radiolabeled DNA fragments (Sambrook et al. 1989) derived from genomic polymerase chain reaction (PCR) products or cloned fragments containing the desired gene. Expression of 3-ketoacyl-CoA thiolase ( $3 k t)$ was analyzed using labeled cDNA from expressed sequence tag clone c3a03a1.r1. The foxA probe was generated using the cloned gene, as previously described (Maggio-Hall and Keller 2004). aflC (formerly norA) expression (GenBank accession number APU24698) was determined by labeling a PCR fragment of the gene generated by primers norAF (ATGGTTCTCCCTACT) and norAR (TCAT TTTGAGGCAGAA). stc $U$ expression was analyzed by labeling the SacII-KpnI fragment of pRB7 (Hicks et al. 1997).

Catalase assays. Cell-free extracts were generated as previously described (Shimizu et al. 2003). Catalase activity was measured as described (Beers and Sizer 1952).

Fluorescence microscopy. Visualization of GFP and NOR was accomplished through the use of an Olympus BX60 microscope fitted with fluorescein isothiocyanate and tetramethylrhodamine isothiocyanate filters, respectively. Images were saved using a Magnafire digital camera and software (Optronics, Goleta, CA, U.S.A.). Best results for A. nidulans strains carrying the $a c u D:$ GFP fusion were obtained by inoculating conidial suspensions $\left(10^{5}\right.$ spores per $\left.\mu \mathrm{l}\right)$ at the base of small cubes of solid medium mounted on sterile glass microscope slides. After the appropriate incubation period ( $24 \mathrm{~h}$ for GFP, 48 to $72 \mathrm{~h}$ for NOR), the medium was removed with sterile forceps, and a drop of $\mathrm{ddH}_{2} \mathrm{O}$ and a cover slip were added. For A. parasiticus and A. flavus strains, mycelium was removed from liquid shake cultures of YES and YES + Tween $80(1 \%$ $\mathrm{vol} / \mathrm{vol}$ ) media at the appropriate time.

\section{ACKNOWLEDGMENTS}

L. A. Maggio-Hall was supported by a National Institute of Health postdoctoral fellowship (F32 AI052654) and R. A. Wilson by National Resources Institute grant 2001-35319-10996 to N. P. Keller. LC/MS quantification of ST was performed by R. Plattner (United States Department of Agriculture-Agricultural Research Service, Peoria, IL).

\section{LITERATURE CITED}

Abdollahi, A., and Buchanan, R. L.1981. Regulation of aflatoxin biosynthesis: Characterization of glucose as an apparent inducer of aflatoxin production. J. Food Sci. 46:143-146.

Askenazi, M., Driggers, E. M. Holtzman, D. A., Norman, T. C., Iverson, S., Zimmer, D. P., Boers, M. E., Blomquist, P. R., Martinez, E. J., Monreal, A. W., Feibelman, T. P., Mayorga, M. E., Maxon, M. E., Sykes, K., Tobin, J. V., Cordero, E., Salama, S. R., Trueheart, J., Royer, J. C., and Madden, K. T. 2003. Integrating transcriptional and metabolite profiles to direct the engineering of lovastatin-producing fungal strains. Nature Biotech. 21:150-156.
Barnes, S. E., Dola, T. P., Bennett, J. W., and Bhatnagar, D. 1994. Synthesis of sterigmatocystin on a chemically defined medium by species of Aspergillus and Chaetomium. Mycopathologia 125:173-178.

Beers, R. F., and Sizer, I. W. 1952. A spectrophotometric method for measuring the breakdown of hydrogen peroxide by catalase. J. Biol. Chem. 195:133-140.

Bosch, F. X., and Peers, F. 1991. Aflatoxins: Data on human carcinogenic risk. IARC Sci. Publ. 48:48-53.

Brown, D. W., Adams, T. H., and Keller, N. P. 1996a. Aspergillus has distinct fatty acid synthases for primary and secondary metabolism. Proc. Natl. Acad. Sci. U.S.A. 93:14873-14877.

Brown, D. W., Yu, J.-H., Kelkar, H., Fernandes, M., Nesbitt, T. C., Keller, N. P., Adams, T. H., and Leonard, T. J. 1996b. Twenty-five co-regulated transcripts define the sterigmatocystin gene cluster in Aspergillus nidulans. Proc. Natl. Acad. Sci. U.S.A. 93:1418-1422.

Brown, R. L., Cotty, P. J., Cleveland, T. E., and Widstrom, N. 1993. Living maize embryo influences accumulation of aflatoxin in maize kernels. J. Food Protect. 56:967-971.

Buchanan, R. L., and Lewis, D. F. 1984. Regulation of aflatoxin biosynthesis: Effect of glucose on activities of various glycolytic enzymes. Appl. Environ. Microbiol. 48:306-310.

Buchanan, R. L., Jones, S. B., Gerasimowicz, W. V., Zaika, L. L., Stahl, H. G., and Ocker. L. A. 1987. Regulation of aflatoxin biosynthesis: Assessment of the role of cellular energy status as a regulator of the induction of aflatoxin production. Appl. Environ. Microbiol. 53:1224-1231.

Burow, G. B., Nesbitt, T. C., Dunlap, J. D., and Keller, N. P. 1997. Seed lipoxygenase products modulate Aspergillus mycotoxin biosynthesis. Mol. Plant-Microbe Interact. 10:380-387.

Burow, G. B., Gardner, H. W., and Keller, N. P. 2000. A peanut seed lipoxygenase responsive to Aspergillus colonization. Plant Mol. Biol. 42:689-701.

Calvo, A. M., Gardner, H. W., and Keller. N. P. 2001. Genetic connection between fatty acid metabolism and sporulation in Aspergillus nidulans. J. Biol. Chem. 276:25766-25774.

Centers for Disease Control and Prevention. 2004. Outbreak of aflatoxin poisoning-Eastern and central provinces, Kenya, 2004. Morb. Mortal. Wkly. Rep. 53:790-793.

Choi, J. K., Yu, F., Wurtele, E. S., and Nikolau, B. J. 1995. Molecular cloning and characterization of the cDNA coding for the biotin-containing subunit of the chloroplastic acetyl-coenzyme A carboxylase. Plant Physiol. 109:619-625.

Davis, N. D., and Diener, U. L. 1968. Growth and aflatoxin production by Aspergillus paraciticus from various carbon sources. Appl. Microbiol. 16:158-159.

Dickman, M. B., Ha, Y.-S., Yang, Z., Adams, B., and Huang, C. 2003. A protein kinase from Colletotrichum trifolii is induced by plant cutin and is required for appressorium formation. Mol. Plant-Microbe Interact. 16:411-421.

Eddine, A. N., Hannemann, F., and Schafer, W. 2001. Cloning and expression analysis of $N h L 1$, a gene encoding an extracellular lipase from the fungal pea pathogen Nectria haematococca MP VI (Fusarium solani f. sp. pisi) that is expressed in planta. Mol. Genet. Genomics 265:215224.

Fabbri, A. A., Fanelli, C., Panfili, G., and Passi, S. 1983. Lipoperoxidation and aflatoxin biosynthesis by Aspergillus parasiticus and A. flavus. J. Gen. Microbiol. 129:3447-3452.

Fanelli, C., Fabbri, A. A., Finotti, E., and Passi, S. 1983. Stimulation of aflatoxin biosynthesis by lipophilic epoxides. J. Gen. Microbiol. 129:1721-1723.

Goodrich-Tanrikulu, M., Mahoney, N., and Rodriguez, S. B. 1995. The plant growth regulator methyl jasmonate inhibits aflatoxin production by Aspergillus flavus. Microbiology. 141:2831-2837.

Hasslacher, M., Ivessa, A. S., Paltauf, F., and Kohlwein, S. D. 1993. Acetyl-CoA carboxylase from yeast is an essential enzyme and is regulated by factors that control phospholipid metabolism. J. Biol. Chem. 268:10946-10952.

Hicks, J. K., Yu, J.-H., Keller, N. P., and Adams, T. H. 1997. Aspergillus sporulation and mycotoxin production both require inactivation of the FadA G $\alpha$ protein-dependent signaling pathway. EMBO (Eur. Mol. Biol. Organ.) J. 16:4916-4923.

Hicks, J. K., Shimizu, K., and Keller, N. P. 2002. Genetics and biosynthesis of aflatoxins and sterigmatocystin. Pages 55-69 in: The Mycota XI. F. Kempken, ed. Springer-Verlag, Berlin.

Hoch, H. C., Staples, R. C., Whitehead, B., Comeau, J., and Wolf, E. D. 1987. Signaling for growth orientation and cell differentiation by surface topography in Uromyces. Science 235:1659-1662.

Hopwood, D. A., and Sherman, D. H. 1990. Molecular genetics of polyketides and its comparison to fatty acid biosynthesis. Annu. Rev. Genet. 24:37-66. 
Howard, R. J., and Valent, B. 1996. Breaking and entering: Host penetration by the fungal rice blast pathogen Magnaporthe grisea. Annu. Rev. Microbiol. 50:491-512.

Hwang, C. S., Flaishman, M. A., and Kolattukudy, P. E. 1995. Cloning of a gene expressed during appressorium formation by Colletotrichum gloeosporioides and a marked decrease in virulence by disruption of this gene. Plant Cell 7:183-193.

Jellum, M. D. 1970. Plant introductions of maize as a source of oil with unusual fatty acid composition. J. Agric. Food Chem. 18:365-370.

Kaefer, E. 1977. Meiotic and mitotic recombination in Aspergillus and its chromosomal aberrations. Pages 33-131 in: Advances in Genetics. E. W. Caspari, ed. Academic Press, London.

Keller, N. P., and Hohn, T. M. 1996. Metabolic pathway gene clusters in filamentous fungi. Fungal Genet. Biol. 21:17-29.

Keller, N. P., Butchko, R. A. E., Sarr, B., and Phillips, T. D. 1994. A visual pattern of mycotoxin production in maize kernels by Aspergillus spp. Phytopathology 84:483-488.

Klein, A. T., van den Berg, M., Bottger, G., Tabak, H. F., and Distel, B. 2002. Saccharomyces cerevisiae acyl-CoA oxidase follows a novel, non-PTS1, import pathway into peroxisomes that is dependent on Pex5p. J. Biol. Chem. 277:25011-25019.

Lancaster, M. D., Jenkins, F. P., and Phillip, J. M. 1961. Toxicity associated with certain samples of groundnuts. Nature 192:1095-1096.

Lee, L. W., Chiou, C. H., Klomparens, K. L., Cary, J. W., and Linz, J. E. 2004. Subcellular localization of aflatoxin biosynthetic enzymes Nor-1, Ver-1, and OmtA in time-dependent fractionated colonies of Aspergillus parasiticus. Arch. Microbiol. 181:204-214.

López-Casillas, F., Bai, D. H., Luo, X. C., Kong, I. S., Hermodson, M. A., and Kim, K. H. 1988. Structure of the coding sequence and primary amino acid sequence of acetyl-coenzyme A carboxylase. Proc. Natl. Acad. Sci. U.S.A. 85:5784-5788.

Maggio-Hall, L. A., and Keller, N. P. 2004. Mitochondrial $\beta$-oxidation in Aspergillus nidulans. Mol. Microbiol. 54:1173-1185.

Mellon, J. E., Cotty, P. J., and Dowd, M. K. 2000. Influence of lipids with and without other cottonseed reserve materials on aflatoxin B1 production by Aspergillus flavus. J. Agric. Food Chem. 48:3611-3615.

Morrice, J., MacKenzie, D. A., Parr, A. J., and Archer, D. B. 1998. Isolation and characterisation of the acetyl-CoA carboxylase gene from Aspergillus nidulans. Curr. Genet. 34:379-385.

Muller, W. H., van der Krift, T. P., Krouwer, A. J., Wosten, H. A., van der Voort, L. H., Smaal, E. B., and Verkleij, A. J. 1991. Localization of the pathway of the penicillin biosynthesis in Penicillium chrysogenum. EMBO (Eur. Mol. Biol. Organ.) J. 10:489-495.

Nemec, T., and Jernejc, K. 2002. Influence of Tween 80 on lipid metabolism of an Aspergillus niger strain. Appl. Biochem. Biotechnol. 101:229-238.

Proctor, R. H., Hohn, T. M., and McCormick, S. P. 1995. Reduced virulence of Gibberella zeae caused by disruption of a trichothecene toxin biosynthetic gene. Mol. Plant-Microbe Interact. 8:593-601.

Purchase, I. F., and Van Der Watt, J. J. 1970. Carcinogenicity of sterigmatocystin. Food Cosmet. Toxicol. 8:289-295.

Robens, J. 2001. The costs of mycotoxin management to the USA: Management of aflatoxin in the United States. In: Mycotoxins: The Cost of Achieving Food Security and Food Quality. APSNet Features August 2001. Online publication.

Roze, L.V., Miller, M. J., Rarick, M., Mahanti, N., and Linz, J. E. 2004. A novel cAMP-response element, CRE1, modulates expression of nor-1 in Aspergillus parasiticus. J. Biol. Chem. 279:27428-27439.

Sacksteder, K. A., Morrell, J. C., Wanders, R. A., Matalon, R., and Gould, S. J. 1999. $M C D$ encodes peroxisomal and cytoplasmic forms of malonyl-coA decarboxylase and is mutated in malonyl-CoA decarboxylase deficiency. J. Biol. Chem. 274:24461-24468.

Severns, D. E., Clements, M. J., Lambert, R. J., and White, D. G. 2003.
Comparison of Aspergillus ear rot and aflatoxin contamination in grain of high-oil and normal-oil corn hybrids. J. Food Prot. 66:637-643.

Shimizu, K., and Keller, N. P. 2001. Genetic involvement of a cAMP-dependent protein kinase in a $\mathrm{G}$ protein signaling pathway regulating morphological and chemical transitions in Aspergillus nidulans. Genetics 157:591-600.

Shimizu, K., Hicks, J. K., Huang, T. P., and Keller, N. P. 2003. Pka, Ras and RGS protein interactions regulate activity of AflR, a $\mathrm{Zn}$ (II)2Cys6 transcription factor in Aspergillus nidulans. Genetics 165:1095-1104.

Skory, C. D., Chang, P. K., Cary, J., and Linz, J. E. 1992. Isolation and characterization of a gene from Aspergillus parasiticus associated with the conversion of versicolorin A to sterigmatocystin in aflatoxin biosynthesis. Appl. Environ. Microbiol. 58:3527-3537.

Smart, M. G., Wicklow, D. T., and Caldwell, R. W. 1990. Pathogenesis in Aspergillus ear rot of maize: Light microscopy of fungal spread from wounds. Phytopathology 80:1287-1294.

Tanaka, A., Osumi, M., and Fukui, S. 1982. Peroxisomes of alkane-grown yeast: Fundamental and practical aspects. Ann. NY Acad. Sci. 386:183199.

Valenciano S., De Lucas, J. R., Pedregosa, A., Monistrol, I. F., and Laborda, F. 1996. Induction of $\beta$-oxidation enzymes and microbody proliferation in Aspergillus nidulans. Arch. Microbiol. 166:336-341.

Valenciano, S., De Lucas, J. R., Van der Klei, I., Veenhuis, M., and Laborda, F. 1998. Characterization of Aspergillus nidulans peroxisomes by immunoelectron microscopy. Arch. Microbiol. 170:370-376.

Veenhuis, M., Mateblowski, M., Kunau, W. H., and Harder, W. 1987. Proliferation of microbodies in Saccharomyces cerevisiae. Yeast 3:77-84.

Watanabe, C. M. H., and Townsend, C. A. 2002. Initial characterization of a Type I fatty acid synthase and polyketide synthase multienzyme complex NorS in the biosynthesis of aflatoxin $\mathrm{B}_{1}$. Chem. Biol. 9:981-988.

Watson, S. A. 1987. Structure and composition. Pages 53-82 in: Corn: Chemistry and Technology. S. A. Watson and P. E. Ramstad, eds. American Association of Cereal Chemists, St. Paul, MN, U.S.A.

Weeradechapon, K. 2002. Enzyme targeting and structural studies on Aspergillus nidulans isocitrate lyase. Ph.D., University of Sheffield, Sheffield, U.K.

Wilson, R. A., Calvo, A. M., Chang, P. K., and Keller, N. P. 2004. Characterization of the Aspergillus parasiticus delta12-desaturase gene: A role for lipid metabolism in the Aspergillus-seed interaction. Microbiology 150:2881-2888.

Wiseman, D. W., and Buchanan, R. L. 1987. Determination of glucose level needed to induce aflatoxin in Aspergillus parasiticus. Can. J. Microbiol. 33:828-830.

Wogan, G. N., Househam, K. C., and Hundt, H. K. 1992. Aflatoxins as risk factors for hepatocellular carcinoma in humans: Aflatoxin exposure and its relationship to kwashiorkor in African children. Cancer Res. 52:2114s-2118s

Xu, J., Nakamura, M. T., Cho, H. P., and Clarke, S. D. 1999. Sterol regulatory element binding protein-1 expression is suppressed by dietary polyunsaturated fatty acids. A mechanism for the coordinate suppression of lipogenic genes by polyunsaturated fats. J. Biol. Chem. 274:23577-23583.

Xue, H. Q., Isleib, T. G., Payne, G. A., Wilson, R. F., Novitzky, W. P., and O'Brian, G. 2003. Comparison of aflatoxin production in normal- and high-oleic backcross-derived peanut lines. Plant Dis. 87:1360-1365.

Yu, J., Mohawed, S. M., Bhatnagar, D., and Cleveland, T. E. 2003. Substrate-induced lipase gene expression and aflatoxin production in Aspergillus parasiticus and Aspergillus flavus. J. Appl. Microbiol. 95:1334-1342.

Zhang, Y. Q., and Keller, N. P. 2004. Blockage of methylcitrate cycle inhibits polyketide production in Aspergillus nidulans. Mol. Microbiol. $52: 541-550$. 